\title{
Spike Controls for Elliptic and Parabolic PDE
}

\author{
E. $\operatorname{Casas}^{\mathrm{a}, 1, *}$, E. Zuazua ${ }^{\mathrm{b}, \mathrm{c}, 2}$ \\ ${ }^{a}$ Departamento de Matemática Aplicada y Ciencias de la Computación, E.T.S.I. \\ Industriales y de Telecomunicación, Universidad de Cantabria, 39005 Santander, Spain \\ ${ }^{b}$ Basque Center for Applied Mathematics (BCAM), Alameda Mazarredo 14, E-48009 \\ Bilbao, Basque Country, Spain \\ ${ }^{c}$ IKERBASQUE, Basque Foundation for Science, E-48011 Bilbao, Basque Country, Spain
}

\begin{abstract}
We analyze the use of measures of minimal norm to control elliptic and parabolic equations. We prove the sparsity of the optimal control. In the parabolic case, we prove that the solution of the optimization problem is a Borel measure supported in a set of null Lebesgue measure. In both cases, the approximate controllability can be achieved efficiently by means of controls that are activated in some finite number of pointwise locations. We also analyze the corresponding dual problem.
\end{abstract}

Keywords: parabolic equations, elliptic equations, approximate controllability, spike controls, Borel measures

2010 MSC: 35J15, 35K15, 49K20, 93B05, 93C20

\section{Introduction}

In this paper we address the issue of controlling elliptic and parabolic equations by means of sparse controls. As we shall see, when looking for the control of minimal measure the sparsity is ensured. This is in contrast with the fact that controls of minimal $L^{2}$ norm end up being smooth and distributed everywhere on the support of the controller while controls of minimal $L^{\infty}$-norm are of bang-bang form (see [1]).

\footnotetext{
* Corresponding author

Email addresses: eduardo.casas@unican.es (E. Casas), zuazua@bcamath.org

(E. Zuazua)

${ }^{1}$ This author was supported by Spanish Ministerio de Economía y Competitividad under project MTM2011-22711.

${ }^{2}$ This author was supported by the ERC Advanced Grant FP7-246775 NUMERIWAVES, the Grant PI2010-04 of the Basque Government, the ESF Research Networking Programme OPTPDE and Grant MTM2011-29306 of the Spanish Ministerio de Economía y Competitividad.
} 
We first analyze the problem of approximate controllability for the heat equation. More precisely, we consider the parabolic equation

$$
\left\{\begin{array}{rlll}
y^{\prime}-\Delta y & = & & \text { in } Q=\Omega \times(0, T), \\
y & =0 & & \text { on } \Sigma=\Gamma \times(0, T), \\
y(0) & =y_{0} & & \text { in } \Omega
\end{array}\right.
$$

where $y_{0} \in L^{2}(\Omega)$ is fixed, $\Omega \subset \mathbb{R}^{n}$ is an open connected bounded set and $\Gamma$ is the boundary of $\Omega$, that we will assume to be Lipschitz.

We wish to choose the control $u$ such that the associated state at time $T$, $y_{u}(T)$, is in the $L^{2}(\Omega)$-ball $\bar{B}_{\varepsilon}\left(y_{d}\right)$, where $y_{d}$ represents the desired final state and $\epsilon>0$ the admissible distance to the target.

It is well known that for any $\epsilon>0$ it is possible to find $u \in L^{2}(Q)$ such that $y_{u}(T) \in \bar{B}_{\varepsilon}\left(y_{d}\right)$; see Lions [2]. In fact the same holds when the control $u$ has its support in a subset $\omega$ of $\Omega$ of positive measure.

We are interested on building and analyzing the structure of the controls $u$ of minimal energy. In the $L^{2}$-setting this can be done by considering the following minimization problem

$$
\min _{y_{u}(T) \in \bar{B}_{\varepsilon}\left(y_{d}\right)} J(u)=\frac{1}{2}\|u\|_{L^{2}(Q)}^{2} .
$$

It can be checked that this problem has a unique solution that is given by $\bar{u}=-\bar{\varphi}$, where $\bar{\varphi}$ is the unique solution of the adjoint equation

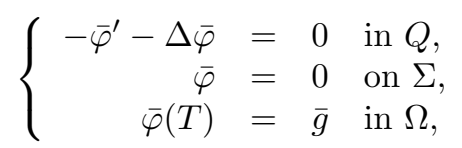

for some $\bar{g} \in L^{2}(\Omega)$ satisfying

$$
\int_{\Omega} \bar{g}(x)(y(x)-\bar{y}(x, T)) d x \leq 0 \quad \forall y \in \bar{B}_{\varepsilon}\left(y_{d}\right) .
$$

Above, $\bar{y}$ denotes the state associated to $\bar{u}$. This means that the control is smooth but active at almost every point of $\Omega$ and at all instant $t$. This makes these controls to be of little practical use in applications where one looks for controls with small support.

In some recent papers, the use of the $L^{1}$-norm instead of the $L^{2}$-norm was shown to be very efficient to obtain optimal controls with support in small regions of the domain, the domain being adjustable in terms of the tuning of suitable parameters entering in the cost functional; see [3], [4], [5], or [6].

However, the above control problem has no solution, in general, if we replace the $L^{2}(Q)$-norm by the $L^{1}(Q)$-norm, i.e. if we take $J(u)=\|u\|_{L^{1}(Q)}$. To overcome this difficulty, the use of Borel measures in $Q$ and the cost functional $J(u)=\|u\|_{M(Q)}$ was suggested in [7]. The supports of the optimal measures turn out to be small. 
In fact, as we shall show, in the present context of the approximate controllability of the heat equation, the optimal measure $\bar{u}$ has a support of null Lebesgue measure. To be more precise, we will prove that given any time interval $\left[T_{0}, T_{1}\right]$, where $0<T_{0}<T_{1}<T$, such that the controls are supported in $\left[T_{0}, T_{1}\right]$, then there exists a measure $\bar{u}_{\Omega} \in M(\Omega)$, having a support of null Lebesgue measure, such that setting $\bar{u}=\bar{u}_{\Omega} \otimes \delta_{T_{1}}$, the property $y_{\bar{u}}(T) \in \bar{B}_{\varepsilon}\left(y_{d}\right)$ holds. Here $\delta_{T_{1}}$ stands for the Dirac measure concentrated at $t=T_{1}$. This shows that, in particular, the optimal measure is concentrated on a set of points of $\Omega$, with zero Lebesgue measure, at the final time instant $T_{1}$. We also prove the uniqueness of this optimal measure. These are the main contributions of this paper.

Hereafter, we will denote by $\bar{u}_{\Omega} \otimes \delta_{T_{1}}$, the measure in $Q$ defined by

$$
\left\langle\bar{u}_{\Omega} \otimes \delta_{T_{1}}, y\right\rangle=\int_{\Omega} y\left(x, T_{1}\right) d \bar{u}_{\Omega}(x) .
$$

As proved in [8], a measure in $\Omega$ can be efficiently approximated by a combination of Dirac measures. As a consequence, we deduce that the approximate controllability can be achieved by activating the controllers in some finite number of pointwise locations at the time $T_{1}$.

On the other hand, from the point of view of applications, it is natural to limit a priori the area where the controls can be placed. Thus, given a (possibly small) region $\omega \subset \Omega$, that we will assume to be an open non-empty set with finitely many connected components, we assume that the support of $u$ is required to be in $\bar{\omega}$.

This leads to the study of the following optimal control problem

$$
(\mathrm{P})\left\{\begin{array}{l}
\min J(u)=\|u\|_{M\left(Q_{0}\right)}, \\
\left(u, y_{u}(T)\right) \in M\left(Q_{0}\right) \times \bar{B}_{\varepsilon}\left(y_{d}\right),
\end{array}\right.
$$

where $Q_{0}=(\Omega \cap \bar{\omega}) \times\left[T_{0}, T_{1}\right], M\left(Q_{0}\right)$ being the space of real and regular Borel measures in $Q_{0}$ and $y_{u}$ the solution of

$$
\left\{\begin{array}{rlll}
y^{\prime}-\Delta y & = & & \text { in } Q \\
y & = & 0 & \text { on } \Sigma \\
y(0) & = & 0 & \text { in } \Omega .
\end{array}\right.
$$

Without loss of generality, we have taken the initial state $y_{0}=0$. Indeed, for $y_{0} \neq 0$ we can consider the solution $\tilde{y}$ of the parabolic equation corresponding to $u=0$ and change $y_{d}$ by $y_{d}-\tilde{y}(T)$. Then the problem is formulated as above. In (1), $u$ is extended by zero outside $Q_{0}$.

To avoid the trivial case where the optimal solution is $\bar{u}=0$, hereafter we will assume that $\left\|y_{d}\right\|_{L^{2}(\Omega)}>\varepsilon$. On the other hand, let us observe that the choice $T_{1}<T$ can be convenient not only for practical reasons, but it is theoretically necessary as well. Indeed, if $T_{1}$ is taken equal to $T$, then $y_{u}(T)$ does not belong, in general, to $L^{2}(\Omega)$. Therefore, the problem $(\mathrm{P})$ is not well 
posed in the indicated spaces. We also take $T_{0}>0$ to avoid the use of (measure) controls as initial condition.

We will denote

$$
C_{0}\left(Q_{0}\right)=\left\{y \in C\left(\bar{Q}_{0}\right): y(x, t)=0 \text { on } \Sigma \cap\left(\partial \omega \times\left[T_{0}, T_{1}\right]\right)\right\} .
$$

Endowed with the maximum norm, this is a Banach space and, according to the Riesz representation theorem (see, for instance, Rudin [9, Theorem 6.19]), $M\left(Q_{0}\right)$ is identified with the dual of $C_{0}\left(Q_{0}\right)$ and

$$
\|u\|_{M\left(Q_{0}\right)}=|u|\left(Q_{0}\right)=\sup _{y \in C_{0}\left(Q_{0}\right),\|y\|_{\infty} \leq 1} \int_{Q_{0}} y(x, t) d u(x, t),
$$

where $|u|$ denotes the total variation measure associated to $u$.

The plan of the paper is as follows. In the next section, we analyze the control problem $(\mathrm{P})$ : we prove the existence and uniqueness of a solution, we get the optimality conditions and establish the spike structure of the optimal control. The dual problem $\left(\mathrm{P}^{*}\right)$ is studied in $\S 3$. Some equivalent formulations for $(\mathrm{P})$ and $\left(\mathrm{P}^{*}\right)$ are considered in $\S 4$. As claimed in [7], convex duality is a powerful framework for solving non-smooth optimal control problems. This has motivated us to consider the study of the dual problems. Finally, the previous results are extended to the elliptic case in $\S 5$.

\section{Analysis of the Control Problem (P)}

Before analyzing the control problem $(\mathrm{P})$, we will comment some known facts about the equation (1). First, we give a definition of solution of (1) and then we study the existence, uniqueness and continuity with respect to the measure $u$; see [10] or [11] for more details.

Definition 1. Given $p, r \in[1,2)$, with $(2 / r)+(n / p)>n+1$, we will say that a function $y \in L^{r}\left([0, T], W_{0}^{1, p}(\Omega)\right)$ is a solution of $(1)$ if the following identity holds

$$
\int_{Q}\left(-\phi^{\prime} y+\nabla \phi \nabla y\right) d x d t=\int_{Q_{0}} \phi d u \quad \forall \phi \in \Phi,
$$

where

$$
\Phi=\left\{\phi \in C^{1}(\bar{Q}): \phi(x, T)=0 \text { in } \Omega \text { and } \phi(x, t)=0 \text { on } \Sigma\right\}
$$

Theorem 1. There exists a unique function $y \in L^{r}\left([0, T], W_{0}^{1, p}(\Omega)\right)$ for all $p, r \in[1,2)$, with $(2 / r)+(n / p)>n+1$, such that it is a solution of (1) and

$$
\int_{Q}\left(-\phi^{\prime}-\Delta \phi\right) y d x d t=\int_{Q_{0}} \phi(x, t) d u(x, t) \quad \forall \phi \in \Phi_{\infty},
$$

where $\Phi_{\infty}=\left\{\phi \in \Phi: \phi^{\prime}+\Delta \phi \in L^{\infty}(Q)\right\}$. Moreover, there exists a constant $C_{r, p}>0$ independent of $u$ such that

$$
\|y\|_{L^{r}\left([0, T], W_{0}^{1, p}(\Omega)\right)}+\|y(T)\|_{L^{2}(\Omega)} \leq C_{r, p}\|u\|_{M\left(Q_{0}\right)} .
$$


ProOF. Let us take a sequence of functions $\left\{u_{k}\right\}_{k=1}^{\infty} \subset C(\bar{Q})$ such that $u_{k} \rightarrow u$ weakly* in $M(Q)$. We can assume that $\operatorname{supp}\left(u_{k}\right) \subset \bar{Q}_{\rho}=\bar{\Omega} \times\left[0, T_{1}+\rho\right]$, with $T_{1}+\rho<T$, and $\left\|u_{k}\right\|_{L^{1}(Q)} \leq\|u\|_{M\left(Q_{0}\right)}$. The standard way to get this sequence is making the convolution of $u$ with a sequence of mollifiers. Now, we take $y_{k} \in L^{2}\left([0, T], H_{0}^{1}(\Omega)\right) \cap C\left([0, T], L^{2}(\Omega)\right)$ solution of

$$
\left\{\begin{aligned}
y_{k}^{\prime}-\Delta y_{k} & =u_{k} & & \text { in } Q \\
y_{k} & =0 & & \text { on } \Sigma \\
y_{k}(0) & =0 & & \text { in } \Omega
\end{aligned}\right.
$$

Then, we can argue as in [10, Theorem 6.3$]$ to deduce that

$$
\left\|y_{k}\right\|_{L^{r}\left([0, T], W_{0}^{1, p}(\Omega)\right)} \leq C_{r, p}\|u\|_{M\left(Q_{0}\right)}
$$

for some constant independent of $k$. As in [10], we get for a subsequence that $y_{k} \rightarrow y$ in $L^{r}\left([0, T], W_{0}^{1, p}(\Omega)\right)$, which is the unique solution of (1) satisfying (3). To get the estimate for $y(T)$ in $L^{2}(\Omega)$ we proceed as follows. Given $g \in L^{2}(\Omega)$, take $\varphi_{g} \in L^{2}\left([0, T], H_{0}^{1}(\Omega)\right) \cap C\left([0, T], L^{2}(\Omega)\right)$ solution of

$$
\left\{\begin{array}{rlll}
\phi^{\prime}+\Delta \phi & = & & \text { in } Q \\
\phi & = & 0 & \text { on } \Sigma \\
\phi(T) & = & g & \text { in } \Omega
\end{array}\right.
$$

Then, we have that $\varphi_{g} \in C\left(\bar{Q}_{\rho}\right)$ and

$$
\left\|\varphi_{g}\right\|_{C\left(\bar{Q}_{\rho}\right)} \leq C_{\rho}\|g\|_{L^{2}(\Omega)}
$$

where $C_{\rho}$ is uniformly bounded for $\rho \leq\left|T-T_{1}\right| / 2$; see [13]. Hence,

$$
\begin{aligned}
& \int_{\Omega} g(x) y_{k}(x, T) d x=\int_{\Omega} \varphi_{g}(x, T) y_{k}(x, T) d x=\int_{Q}\left(y_{k}^{\prime}-\Delta y_{k}\right) \varphi_{g} d x d t \\
& =\int_{Q} u_{k}(x, t) \varphi_{g}(x, t) d x d t \leq\left\|u_{k}\right\|_{L^{1}\left(Q_{\rho}\right)}\left\|\varphi_{g}\right\|_{C\left(\bar{Q}_{\rho}\right)} \leq C_{\rho}\|u\|_{M\left(Q_{0}\right)}\|g\|_{L^{2}(\Omega)} .
\end{aligned}
$$

Since $g \in L^{2}(\Omega)$ is arbitrary, we deduce the estimate for $\left\|y_{k}(T)\right\|_{L^{2}(\Omega)}$, which implies the desired estimate for $y(T)$ in $L^{2}(\Omega)$.

Remark 1. Let us note that passing to the limit as $k \rightarrow \infty$ in the last relations of the previous proof, we obtain

$$
\int_{\Omega} g(x) y_{u}(x, T) d x=\int_{Q_{0}} \varphi_{g}(x, t) d u \quad \forall u \in M\left(Q_{0}\right) \quad \text { and } \forall g \in L^{2}(\Omega),
$$

where $y_{u}$ and $\varphi_{g}$ are the solutions of (1) and (6), respectively.

Now, we return to the control problem $(\mathrm{P})$. We observe that $(\mathrm{P})$ is a convex problem, but the cost functional $J$ is not strictly convex. Despite of this the optimal control is unique as the following result shows. 
Theorem 2. The control problem (P) has at least one solution $\bar{u}$. Moreover, there exists a unique element $\bar{y}_{T} \in L^{2}(\Omega)$, with $\left\|\bar{y}_{T}-y_{d}\right\|_{L^{2}(\Omega)}=\varepsilon$, such that $y_{u}(T)=\bar{y}_{T}$ for every solution $u$ of $(\mathrm{P})$.

Proof. First of all, we prove that the set of feasible controls is non-empty for any $\varepsilon>0$. From [12] we deduce the existence of an element $y_{1} \in L^{2}(\Omega)$ such that the solution $\hat{y}$ of the problem

$$
\left\{\begin{array}{rlll}
y^{\prime}-\Delta y & =0 & & \text { in } \Omega \times\left(T_{1}, T\right), \\
y & =0 & & \text { on } \Gamma \times\left(T_{1}, T\right), \\
y\left(T_{1}\right) & =y_{1} & & \text { in } \Omega,
\end{array}\right.
$$

satisfies $\left\|\hat{y}(T)-y_{d}\right\|_{L^{2}(\Omega)}<\frac{\varepsilon}{2}$. On the other hand, due to the approximate controllability property of the heat equation (see Lions [2]), we have that there exists an element $u \in L^{2}\left(Q_{0}\right)$ such that $\left\|y_{u}\left(T_{1}\right)-y_{1}\right\|_{L^{2}(\Omega)}<\frac{\varepsilon}{2}$. Then we have

$$
\begin{gathered}
\left\|y_{u}(T)-y_{d}\right\|_{L^{2}(\Omega)} \leq\left\|y_{u}(T)-\hat{y}(T)\right\|_{L^{2}(\Omega)}+\left\|\hat{y}(T)-y_{d}\right\|_{L^{2}(\Omega)} \\
\leq\left\|y_{u}\left(T_{1}\right)-y_{1}\right\|_{L^{2}(\Omega)}+\left\|\hat{y}(T)-y_{d}\right\|_{L^{2}(\Omega)}<\varepsilon
\end{gathered}
$$

Since $L^{2}\left(Q_{0}\right)$ is identified with a subspace of $M\left(Q_{0}\right)$, we conclude the desired result.

Now, the existence of a solution can be easily proved by taking a minimizing sequence and using the continuity of $u \in M\left(Q_{0}\right) \rightarrow y_{u}(T) \in L^{2}(\Omega)$ established in Theorem 1. On the other hand, if $u \in M\left(Q_{0}\right)$ and $\left\|y_{u}(T)-y_{d}\right\|_{L^{2}(\Omega)}<\varepsilon$, then we can take $0<\lambda<1$ such that

$$
\left\|y_{u}(T)-y_{d}\right\|_{L^{2}(\Omega)}+\lambda\left\|y_{u}(T)\right\|_{L^{2}(\Omega)} \leq \varepsilon .
$$

Setting $u_{\lambda}=(1-\lambda) u$, then $J\left(u_{\lambda}\right)=(1-\lambda) J(u)<J(u)$ and $\| y_{u_{\lambda}}(T)-$ $y_{d} \|_{L^{2}(\Omega)} \leq \varepsilon$, hence $u$ is not a solution of $(\mathrm{P})$. Therefore, to any solution $u$ of (P) corresponds an optimal state $y$ such that $y(T)$ is on the boundary of the ball $\bar{B}_{\varepsilon}\left(y_{d}\right)$.

Finally, let us prove the uniqueness of $\bar{y}_{T}=y_{u}(T)$. We argue by contradiction. Let $u_{1}$ and $u_{2}$ be two different solutions of $(\mathrm{P})$ with two different final states $y_{u_{1}}(T) \neq y_{u_{2}}(T)$. We know that

$$
\left\|y_{u_{1}}(T)-y_{d}\right\|_{L^{2}(\Omega)}=\left\|y_{u_{2}}(T)-y_{d}\right\|_{L^{2}(\Omega)}=\varepsilon .
$$

Define $u=\left(u_{1}+u_{2}\right) / 2$. Using the convexity of $J$ and the strict convexity of the $L^{2}(\Omega)$-norm, we infer

$$
J(u) \leq \frac{1}{2}\left(J\left(u_{1}\right)+J\left(u_{2}\right)\right)=\inf (\mathrm{P}) \text { and }\left\|y_{u}(T)-y_{d}\right\|_{L^{2}(\Omega)}<\varepsilon .
$$

Thus, $u$ is a solution of $(\mathrm{P})$ with $y_{u}(T)$ belonging to the interior of the ball $\bar{B}_{\varepsilon}\left(y_{d}\right)$, which contradicts the property established above for the solutions of (P). This implies the existence of a unique element $\bar{y}_{T}$ satisfying the conditions of the theorem. 
The next step in our analysis is the proof of the optimality conditions.

Theorem 3. Let $\bar{u} \in M\left(Q_{0}\right)$ such that $\bar{y}(T) \in \bar{B}_{\varepsilon}\left(y_{d}\right)$, where $\bar{y}$ is the state associated to $\bar{u}$. Then, $\bar{u}$ is a solution of problem $(\mathrm{P})$ if and only if there exist two unique elements $\bar{g} \in L^{2}(\Omega)$ and $\bar{\varphi} \in L^{2}\left([0, T], H_{0}^{1}(\Omega)\right) \cap C\left([0, T], L^{2}(\Omega)\right)$ such that

$$
\begin{aligned}
& \int_{\Omega} \bar{g}(x)(y(x)-\bar{y}(x, T)) d x \leq 0 \quad \forall y \in \bar{B}_{\varepsilon}\left(y_{d}\right), \\
& \left\{\begin{array}{rlll}
\bar{\varphi}^{\prime}+\Delta \bar{\varphi} & = & & \text { in } Q, \\
\bar{\varphi} & = & 0 & \text { on } \Sigma, \\
\bar{\varphi}(T) & = & \bar{g} & \text { in } \Omega,
\end{array}\right. \\
& \|\bar{u}\|_{M\left(Q_{0}\right)}=-\int_{Q_{0}} \bar{\varphi}(x, t) d \bar{u}, \\
& \|\bar{\varphi}\|_{C\left(Q_{0}\right)}=1 \text {. }
\end{aligned}
$$

Furthermore, there exists a real number $\bar{\lambda}>0$ such that $\bar{g}=\bar{\lambda}\left(\bar{y}(T)-y_{d}\right)$. Finally, $\bar{g}$ and $\bar{\varphi}$ are the same for every solution of $(\mathrm{P})$.

Proof. Let us consider the linear mapping $A \in \mathcal{L}\left(M\left(Q_{0}\right), L^{2}(\Omega)\right)$, defined by $A u=y_{u}(T)$. The continuity of $A$ follows from (4). Now, we define the functional $\mathcal{J}: M\left(Q_{0}\right) \longrightarrow(-\infty,+\infty]$ by

$$
\mathcal{J}(u)=J(u)+I_{\bar{B}_{\varepsilon}\left(y_{d}\right)}(A u),
$$

where $I_{\bar{B}_{\varepsilon}\left(y_{d}\right)}$ denotes the indicator function of the ball $\bar{B}_{\varepsilon}\left(y_{d}\right)$; which means that it vanishes in $\bar{B}_{\varepsilon}\left(y_{d}\right)$ and takes the value $+\infty$ outside. The problem $(\mathrm{P})$ can be reformulated as the minimization of the convex functional $\mathcal{J}$. Then, $\bar{u}$ is a solution of $(\mathrm{P})$ if and only if $0 \in \partial \mathcal{J}(\bar{u})$. Now, we apply the rules of the sub-differential calculus of convex functions; see, for instance, [14, Chapter 1 , $\S 5.3]$. To this end, we take into account that, according to the proof of Theorem 2 , there exists $u_{0} \in M\left(Q_{0}\right)$ such that $A u_{0} \in B_{\varepsilon}\left(y_{d}\right)$, which means that the Slater condition is fulfilled, consequently

$$
0 \in \partial \mathcal{J}(\bar{u}) \subset \partial J(\bar{u})+A^{*} \partial I_{\bar{B}_{\varepsilon}\left(y_{d}\right)}(\bar{y}) .
$$

This implies that there exists $\bar{g} \in \partial I_{\bar{B}_{\varepsilon}\left(y_{d}\right)}(\bar{y})$ such that $-A^{*} \bar{g} \in \partial J(\bar{u})$. Relation (9) is precisely the definition of $\bar{g} \in \partial I_{\bar{B}_{\varepsilon}\left(y_{d}\right)}(\bar{y})$. Now, we take $\bar{\varphi}$ as the solution of (10). Then, from (8) we deduce

$$
\left\langle A^{*} \bar{g}, u\right\rangle=\langle\bar{g}, A u\rangle=\int_{\Omega} \bar{g}(x) y_{u}(x, T) d x=\int_{Q_{0}} \bar{\varphi}(x, t) d u \quad \forall u \in M\left(Q_{0}\right) .
$$

Combining this identity with the definition of $-A^{*} \bar{g} \in \partial J(\bar{u})$

$$
\left\langle-A^{*} \bar{g}, u-\bar{u}\right\rangle+J(\bar{u}) \leq J(u) \quad \forall u \in M\left(Q_{0}\right),
$$


we obtain

$$
\int_{Q_{0}} \bar{\varphi}(x, t) d \bar{u}-\int_{Q_{0}} \bar{\varphi}(x, t) d u+\|\bar{u}\|_{M\left(Q_{0}\right)} \leq\|u\|_{M\left(Q_{0}\right)} \quad \forall u \in M\left(Q_{0}\right) .
$$

Taking $u=2 \bar{u}$ and $\frac{1}{2} \bar{u}$, respectively, in the above inequality, we get (11). Therefore, the above inequality and (11) imply

$$
-\int_{Q_{0}} \bar{\varphi}(x, t) d u \leq\|u\|_{M\left(Q_{0}\right)} \forall u \in M\left(Q_{0}\right) .
$$

For any point $\left(x_{0}, t_{0}\right) \in Q_{0}$ we select $u= \pm \delta_{\left(x_{0}, t_{0}\right)}$ in the above inequality, which shows that $\pm \bar{\varphi}\left(x_{0}, t_{0}\right) \leq 1$. Since $\left(x_{0}, t_{0}\right)$ is arbitrary in $Q_{0}$, we get that $\|\bar{\varphi}\|_{C\left(Q_{0}\right)} \leq 1$. This inequality along with (11) and the fact that $\bar{u} \neq 0$ imply (12).

Finally, we prove the uniqueness of $\bar{g}$, the corresponding uniqueness for $\bar{\varphi}$ being an immediate consequence. From Theorem 2 and (9) it follows

$$
\int_{\Omega} \bar{g}\left(y-y_{d}\right) d x \leq \int_{\Omega} \bar{g}\left(\bar{y}_{T}-y_{d}\right) d x \quad \forall y \in \bar{B}_{\varepsilon}\left(y_{d}\right)
$$

or equivalently

$$
\int_{\Omega} \bar{g} y d x \leq \int_{\Omega} \bar{g}\left(\bar{y}_{T}-y_{d}\right) d x \quad \forall y \in \bar{B}_{\varepsilon}(0),
$$

which implies the existence of some positive number $\bar{\lambda}$ such that $\bar{g}=\bar{\lambda}\left(\bar{y}_{T}-y_{d}\right)$. Observe that $\bar{g} \neq 0$ because $\|\bar{\varphi}\|_{C_{0}\left(Q_{0}\right)}=1$. Moreover, the last identity implies that $\bar{\lambda}$ is uniquely determined, which concludes the proof.

As a consequence of the previous theorem we get the following result about the structure of the optimal measure.

Corollary 1. Let $\bar{u}$ be a solution of $(\mathrm{P})$. Then, there exist two Borel measures $\hat{u} \in M\left([\partial \omega \cap \Omega] \times\left[T_{0}, T_{1}\right]\right)$ and $\bar{u}_{\omega} \in M(\bar{\omega} \cap \Omega)$ such that

$$
\bar{u}=\hat{u}+\bar{u}_{\omega} \otimes \delta_{T_{1}}, \quad \text { with }\left|\operatorname{supp}\left(\bar{u}_{\omega}\right)\right|=0,
$$

where $|\cdot|$ denotes the Lebesgue measure in $\mathbb{R}^{n}$. Finally, if $\omega=\Omega$, then $\bar{u}=$ $\bar{u}_{\Omega} \otimes \delta_{T_{1}}$, with $\operatorname{supp}\left(\bar{u}_{\Omega}\right) \subset \Omega$ and $\left|\operatorname{supp}\left(\bar{u}_{\Omega}\right)\right|=0$.

ProOF. Let us consider the Jordan decomposition of the measure $\bar{u}, \bar{u}=\bar{u}^{+}-$ $\bar{u}^{-}$, and denote $Q_{0}^{+}=\operatorname{supp}\left(\bar{u}^{+}\right)$and $Q_{0}^{-}=\operatorname{supp}\left(\bar{u}^{-}\right)$. From (11) and (12) we deduce

$$
\begin{aligned}
& \|\bar{u}\|_{M\left(Q_{0}\right)}=|\bar{u}|\left(Q_{0}\right)=\int_{Q_{0}^{+}} d \bar{u}^{+}(x, t)+\int_{Q_{0}^{-}} d \bar{u}^{-}(x, t) \\
& \geq-\int_{Q_{0}^{+}} \bar{\varphi}(x, t) d \bar{u}^{+}(x, t)+\int_{Q_{0}^{-}} \bar{\varphi}(x, t) d \bar{u}^{-}(x, t) \\
& =-\int_{Q_{0}} \bar{\varphi}(x, t) d \bar{u}(x, t)=\|\bar{u}\|_{M\left(Q_{0}\right)} .
\end{aligned}
$$


Hence,

$$
\int_{Q_{0}^{+}}(1+\bar{\varphi}(x, t)) d \bar{u}^{+}(x, t)+\int_{Q_{0}^{-}}(1-\bar{\varphi}(x, t)) d \bar{u}^{-}(x, t)=0,
$$

therefore

$$
\int_{Q_{0}^{+}}(1+\bar{\varphi}(x, t)) d \bar{u}^{+}(x, t)=\int_{Q_{0}^{-}}(1-\bar{\varphi}(x, t)) d \bar{u}^{-}(x, t)=0 .
$$

These identities imply that

$$
\begin{aligned}
& \operatorname{supp}\left(\bar{u}^{+}\right) \subset\left\{(x, t) \in Q_{0}: \bar{\varphi}(x, t)=-1\right\}, \\
& \operatorname{supp}\left(\bar{u}^{-}\right) \subset\left\{(x, t) \in Q_{0}: \bar{\varphi}(x, t)=+1\right\} .
\end{aligned}
$$

Let us prove that $|\bar{\varphi}(x, t)|<1$ for every $(x, t) \in \omega \times\left[T_{0}, T_{1}\right)$. We first observe that the regularizing property of the heat operator implies that $\bar{\varphi} \in$ $C^{\infty}(\Omega \times(0, T)) \cap C(\bar{\Omega} \times[0, T-\varepsilon])$ for every $\varepsilon>0$. Now, we argue by contradiction. Suppose that there exists a point $\left(x_{0}, t_{0}\right) \in \omega \times\left[T_{0}, T_{1}\right)$ such that $\bar{\varphi}\left(x_{0}, t_{0}\right)=+1$. Then, from (12) and the strong maximum principle (see, for instance, Evans [15, Theorem 11, page 375]) we deduce that $\bar{\varphi}(x, t)=+1$ for every $(x, t) \in$ $\omega_{x_{0}} \times\left[t_{0}, T_{1}\right]$, where $\omega_{x_{0}}$ is the open connected component of $\omega$ containing $x_{0}$. Now, using that the function $x \in \Omega \rightarrow \bar{\varphi}(x, t)$ is analytic for every $t$, we deduce from the principle of analytic that $\bar{\varphi}(x, t)=+1$ for every $x \in \Omega$ and $t \in\left[t_{0}, T_{1}\right]$. However, this contradicts the fact that $\bar{\varphi}(x, t)=0$ for $x \in \Gamma$. Analogously, we can prove that $\bar{\varphi}(x, t)>-1$ for every $(x, t) \in \omega \times\left[T_{0}, T_{1}\right)$. This leads to the structure of $\bar{u}$ described in (13). The identity $\left|\operatorname{supp}\left(\bar{u}_{\omega}\right)\right|=0$ follows from the analyticity of the function $x \in \omega \rightarrow \bar{\varphi}\left(x, T_{1}\right)$. Indeed, the analyticity implies that either the set of points where $\bar{\varphi}\left(x, T_{1}\right)=+1$ has a zero Lebesgue measure or $\bar{\varphi}\left(x, T_{1}\right)=+1$ for every $x \in \Omega$. The last option is not possible because $\bar{\varphi}\left(x, T_{1}\right)=0$ on $\Gamma$. Thus, (13) follows. If $\omega=\Omega$, from the boundary condition $\bar{\varphi}=0$ on $\Sigma$, we conclude that $\hat{u}=0$.

Corollary 2. Let us assume that $\omega=\Omega$. Then, there exists a unique solution $\bar{u}$ of problem $(\mathrm{P})$.

Proof. Let $u_{1}$ and $u_{2}$ be two solutions of $(\mathrm{P})$. Then, from Corollary 1 we deduce the existence of two Borel measures $u_{1 \Omega}, u_{2 \Omega} \in M(\Omega)$ such that $u_{i}=u_{i \Omega} \otimes \delta_{T_{1}}$, $i=1,2$. Let us take $u_{\Omega}=u_{2 \Omega}-u_{1 \Omega}, u=u_{\Omega} \otimes \delta_{T_{1}}$ and $y_{u}=y_{u_{2}}-y_{u_{1}}$. Then $y_{u}$ satisfies (1). From the uniqueness of $\bar{y}_{T}$ established in Theorem 2, we also have that $y_{u}(T)=0$. Now, for every $g \in L^{2}(\Omega)$ let $\varphi_{g}$ be the solution of (6). Then, (8) implies that $\left\langle u_{\Omega}, \varphi_{g}\left(T_{1}\right)\right\rangle=0$ for every $g \in L^{2}(\Omega)$. Since the space $S=\left\{\varphi_{g}\left(T_{1}\right): g \in L^{2}(\Omega)\right\}$ is dense in $L^{2}(\Omega)$ because of the approximate controllability of the heat equation, we obtain that $S$ is also dense in $C_{0}(\Omega)$ and consequently $u_{\Omega}=0$. This concludes the uniqueness proof.

Remark 2. Let us consider the case $n=1$, where $\omega=(a, b)$ is a subinterval of $\Omega=(\alpha, \beta)$. Then, the analyticity of the function $x \in \omega \subset \mathbb{R} \rightarrow \bar{\varphi}\left(x, T_{1}\right)$ 
implies that there exists a finite set of points $\left\{x_{j}\right\}_{j=1}^{N_{0}} \subset \bar{\omega}$ and real numbers $\left\{\bar{\lambda}_{j}\right\}_{j=1}^{N_{0}} \subset \mathbb{R}$ such that

$$
\bar{u}_{\omega}=\sum_{j=1}^{N_{0}} \bar{\lambda}_{j} \delta_{x_{j}}
$$

On the other hand, using the analyticity of the functions $t \in(0, T) \rightarrow \bar{\varphi}(a, t)$ and $t \in(0, T) \rightarrow \bar{\varphi}(b, t)$, we deduce the existence of two finite sets of time instants $\left\{t_{a, j}\right\}_{j=1}^{N_{a}}$ and $\left\{t_{b, j}\right\}_{j=1}^{N_{b}}$ and real numbers $\left\{\bar{\lambda}_{a, j}\right\}_{j=1}^{N_{a}}$ and $\left\{\bar{\lambda}_{b, j}\right\}_{j=1}^{N_{b}}$ such that

$$
\hat{u}=\sum_{j=1}^{N_{a}} \bar{\lambda}_{a, j} \delta_{\left(a, t_{a, j}\right)}+\sum_{j=1}^{N_{b}} \bar{\lambda}_{b, j} \delta_{\left(b, t_{b, j}\right)}
$$

\section{The Dual Problem $\left(\mathbf{P}^{*}\right)$}

In this section we are going to get the expression of the dual problem of $(\mathrm{P})$ in the sense of Fenchel and Rockafellar. To this end, we will follow [14, Chapter $3]$. Then we have to identify two pairs of topological vector spaces $(V, Y)$ and $\left(V^{*}, Y^{*}\right)$ with a duality relation. We will take $V=M\left(Q_{0}\right)$ endowed with the weak*-topology, $V^{*}=C_{0}\left(Q_{0}\right)$ and $Y=Y^{*}=L^{2}(\Omega)$. With the notation introduced in the proof of Theorem 3, we have $A \in \mathcal{L}(V, Y), A u=y_{u}(T)$ and the functional $\mathcal{J}: V \longrightarrow \mathbb{R}$ given by $\mathcal{J}(u)=J(u)+I_{\bar{B}_{\varepsilon}\left(y_{d}\right)}(A u)$. Then, the dual problem is defined by

$$
\left(\mathrm{P}^{*}\right) \sup _{g \in Y^{*}}\left\{-J^{*}\left(A^{*} g\right)-I_{\bar{B}_{\varepsilon}\left(y_{d}\right)}^{*}(-g)\right\},
$$

where $J^{*}$ and $I_{\bar{B}_{\varepsilon}\left(y_{d}\right)}^{*}$ are the Fenchel conjugate functions of $J$ and $I_{\bar{B}_{\varepsilon}\left(y_{d}\right)}$, respectively. Let us to obtain the expression of the above terms. First, we look at $A^{*} g$. Since $A^{*} \in \mathcal{L}\left(Y^{*}, V^{*}\right)=\mathcal{L}\left(L^{2}(\Omega), C_{0}\left(Q_{0}\right)\right)$, for every $u \in M\left(Q_{0}\right)$ we get with $(8)$

$$
\left\langle u, A^{*} g\right\rangle=\langle A u, g\rangle=\int_{\Omega} y_{u}(T) g d x=\int_{Q_{0}} \varphi_{g}(x, t) d u(x, t)=\left\langle u, \varphi_{\left.\right|_{\left.\right|_{0}}}\right\rangle,
$$

where $\varphi_{g}$ is the solution of (10). Notice that $\varphi_{g_{Q_{0}}} \in C_{0}\left(Q_{0}\right)$. Therefore, we deduce that $A^{*} g=\left.\varphi_{g}\right|_{Q_{0}}$. Now, from the definition of a conjugate function we get

$$
\begin{gathered}
J^{*}\left(A^{*} g\right)=\sup _{u \in M\left(Q_{0}\right)}\left\{\left\langle u, A^{*} g\right\rangle-J(u)\right\} \\
=\sup _{u \in M\left(Q_{0}\right)}\left\{\int_{Q_{0}} \varphi_{g}(x, t) d u(x, t)-\|u\|_{M\left(Q_{0}\right)}\right\}=\left\{\begin{array}{cl}
+\infty & \text { if }\left\|\varphi_{g}\right\|_{C_{0}\left(Q_{0}\right)}>1, \\
0 & \text { otherwise. }
\end{array}\right.
\end{gathered}
$$

On the other hand,

$$
I_{\bar{B}_{\varepsilon}\left(y_{d}\right)}^{*}(-g)=\sup _{\psi \in L^{2}(\Omega)}\left\{(\psi,-g)_{L^{2}(\Omega)}-I_{\bar{B}_{\varepsilon}\left(y_{d}\right)}(\psi)\right\}=\sup _{\psi \in \bar{B}_{\varepsilon}\left(y_{d}\right)} \int_{\Omega} \psi g d x
$$




$$
=\sup _{\psi \in \bar{B}_{\varepsilon}(0)} \int_{\Omega}\left(\psi+y_{d}\right) g d x=\varepsilon\|g\|_{L^{2}(\Omega)}+\left(y_{d}, g\right)_{L^{2}(\Omega)} .
$$

From (14) and (15) we obtain the following expression for the dual problem

$$
\left(\mathrm{P}^{*}\right)\left\{\begin{array}{l}
\sup \left\{-\varepsilon\|g\|_{L^{2}(\Omega)}-\left(y_{d}, g\right)_{L^{2}(\Omega)}\right\} \\
\left(g, \varphi_{\left.\right|_{Q_{0}}}\right) \in L^{2}(\Omega) \times B
\end{array}\right.
$$

where $B$ is the unit closed ball of $C_{0}\left(Q_{0}\right)$ centered at 0 . Let us introduce the functions $G: L^{2}(\Omega) \longrightarrow \mathbb{R}$ and $\mathcal{G}: L^{2}(\Omega) \longrightarrow(-\infty,+\infty]$ given by

$$
G(g)=\varepsilon\|g\|_{L^{2}(\Omega)}+\left(y_{d}, g\right)_{L^{2}(\Omega)} \text { and } \mathcal{G}(g)=G(g)+I_{B}\left(A^{*} g\right) .
$$

Now, the problem $\left(\mathrm{P}^{*}\right)$ can be written

$$
\left(\mathrm{P}^{*}\right) \sup _{g \in L^{2}(\Omega)}\{-\mathcal{G}(g)\}=-\inf _{g \in L^{2}(\Omega)} \mathcal{G}(g) .
$$

Finally, we study the relation between $(\mathrm{P})$ and $\left(\mathrm{P}^{*}\right)$. Observe that the existence and uniqueness of a solution for $\left(\mathrm{P}^{*}\right)$ is not obvious a priori. However, this is true as claimed in the next theorem.

Theorem 4. Let $(\bar{u}, \bar{y}, \bar{\varphi}, \bar{g})$ given by Theorem 3. Then, $\bar{g}$ is the unique solution of problem $\left(\mathrm{P}^{*}\right)$ and $\inf (\mathrm{P})=\sup \left(\mathrm{P}^{*}\right)$.

Proof. First, we recall that $\bar{\varphi}_{\left.\right|_{Q_{0}}}=A^{*} \bar{g}$ and $\|\bar{\varphi}\|_{C_{0}\left(Q_{0}\right)}=1$, see $(12)$, hence $\mathcal{G}(\bar{g})<+\infty$. Since $\mathcal{G}$ is a convex function, then $\bar{g}$ is a minimum of $\mathcal{G}$ if and only if $0 \in \partial \mathcal{G}(\bar{g})$. To calculate $\partial \mathcal{G}(\bar{g})$ we use that the Slater condition is fulfilled. Indeed, $A^{*} 0=0$ is in the interior of the unit ball $B$, therefore

$$
\partial \mathcal{G}(\bar{g})=G^{\prime}(\bar{g})+A \partial I_{B}\left(A^{*} \bar{g}\right)=\frac{\varepsilon}{\|\bar{g}\|_{L^{2}(\Omega)}} \bar{g}+y_{d}+A \partial I_{B}(\bar{\varphi}) .
$$

From the expression $\bar{g}=\bar{\lambda}\left(\bar{y}(T)-y_{d}\right)$ obtained in Theorem 3 and the property $\left\|\bar{y}(T)-y_{d}\right\|_{L^{2}(\Omega)}=\varepsilon$ proved in Theorem 2 , we obtain

$$
\partial \mathcal{G}(\bar{g})=\bar{y}(T)+A \partial I_{B}(\bar{\varphi}) .
$$

If we prove that $-\bar{u} \in \partial I_{B}(\bar{\varphi})$, then from the identity $\bar{y}(T)=A \bar{u}$, we conclude that $0 \in \partial \mathcal{G}(\bar{g})$. Thus, $\bar{g}$ is a solution of $\left(\mathrm{P}^{*}\right)$. Let us prove that $-\bar{u} \in \partial I_{B}(\bar{\varphi})$, which means

$$
\langle-\bar{u}, \phi-\bar{\varphi}\rangle \leq 0 \quad \forall \phi \in B \text {. }
$$

This is equivalent to

$$
\sup _{\phi \in B} \int_{Q_{0}} \phi d \bar{u} \leq-\int_{Q_{0}} \bar{\varphi} d \bar{u} \Leftrightarrow\|\bar{u}\|_{M\left(Q_{0}\right)} \leq-\int_{Q_{0}} \bar{\varphi} d \bar{u}
$$

which follows from (11). 
Now, we prove that $\inf (\mathrm{P})=\sup \left(\mathrm{P}^{*}\right)$. Using again that $\bar{g}=\bar{\lambda}\left(\bar{y}(T)-y_{d}\right)$ and $\left\|\bar{y}(T)-y_{d}\right\|_{L^{2}(\Omega)}=\varepsilon$ along with (8) and (11), we get

$$
\begin{aligned}
& \mathcal{G}(\bar{g})=\varepsilon\|\bar{g}\|_{L^{2}(\Omega)}+\left(y_{d}, \bar{g}\right)_{L^{2}(\Omega)}=\bar{\lambda}\left\|\bar{y}(T)-y_{d}\right\|_{L^{2}(\Omega)}^{2}+\bar{\lambda}\left(y_{d}, \bar{y}(T)-y_{d}\right)_{L^{2}(\Omega)} \\
= & \bar{\lambda}\left(\bar{y}(T), \bar{y}(T)-y_{d}\right)_{L^{2}(\Omega)}=(\bar{y}(T), \bar{g})_{L^{2}(\Omega)}=\int_{Q_{0}} \bar{\varphi} d \bar{u}=-\|\bar{u}\|_{M\left(Q_{0}\right)}=-\mathcal{J}(\bar{u}),
\end{aligned}
$$

thus

$$
\sup \left(\mathrm{P}^{*}\right)=-\mathcal{G}(\bar{g})=\mathcal{J}(\bar{u})=\inf (\mathrm{P}) .
$$

Finally, let us prove that $\bar{g}$ is the unique solution of $\left(\mathrm{P}^{*}\right)$. If $g \in L^{2}(\Omega)$ is different from $\delta \bar{g}$ for every $\delta \in \mathbb{R}$, the inequality $\|(g+\bar{g}) / 2\|_{L^{2}(\Omega)}<\left(\|g\|_{L^{2}(\Omega)}+\right.$ $\left.\|g\|_{L^{2}(\Omega)}\right) / 2$ implies that $g$ can not be a solution of $\left(\mathrm{P}^{*}\right)$ because it would lead to a contradiction. If $g=\delta \bar{g}$ for some real number $|\delta|>1$, then $\left\|\varphi_{g}\right\|_{C_{0}\left(Q_{0}\right)}=$ $|\delta|\|\bar{\varphi}\|_{C_{0}\left(Q_{0}\right)}=|\delta|>1$, then $\mathcal{G}(g)=+\infty$, and $g$ is not a solution. If $\delta \leq 0$, then

$$
G(g) \geq\left(y_{d}, g\right)_{L^{2}(\Omega)} \geq \delta G(\bar{g})=-\delta\|\bar{u}\|_{M\left(Q_{0}\right)}>-\|\bar{u}\|_{M\left(Q_{0}\right)}=G(\bar{g}) .
$$

Finally, if $0<\delta<1$, then $G(g)=\delta G(\bar{g})>G(\bar{g})$ due to the fact that $G(\bar{g})<0$. This concludes that $\bar{g}$ is the unique solution of $\left(\mathrm{P}^{*}\right)$.

\section{Some equivalent formulations for $(P)$ and $\left(P^{*}\right)$}

So far we have proved that the system (1) can be approximately controlled by using Borel measures with sparse support. To do this we have followed a direct approach, just looking for the minimum of problem $(\mathrm{P})$. However, the analysis of the approximate controllability of the heat equation has traditionally followed a different approach. Indeed, the approximate controllability of (1) by using $L^{2}$ controls concentrated in $\omega \times(0, T)$ has been obtained by studying the adjoint optimization problem

$$
\left(\mathrm{P}_{\varepsilon}\right) \min _{g \in L^{2}(\Omega)} J_{\varepsilon}(g)=\frac{1}{2} \int_{0}^{T} \int_{\omega}\left|\varphi_{g}\right|^{2} d x d t+\varepsilon\|g\|_{L^{2}(\Omega)}-\int_{\Omega} y_{d} g d x,
$$

where $\varphi_{g}$ is the solution of (6). In [2], it was proved that $\left(\mathrm{P}_{\varepsilon}\right)$ has a unique solution $\bar{g}$. Then, the control $\bar{u} \in L^{2}(\omega \times(0, T))$ of minimum norm, with associated state $\bar{y}$, satisfying that $\left\|\bar{y}(T)-y_{d}\right\|_{L^{2}(\Omega)} \leq \varepsilon$ is given by $\bar{u}=\bar{\varphi} \chi_{\omega \times(0, T)}$, where $\chi_{\omega}$ is the characteristic function of $\omega \times(0, T)$ and $\bar{\varphi}$ is the solution of $(6)$ corresponding to $\bar{g}$.

Later, the problem of controlling approximately the system (1) by using bang-bang controls was studied in [16]. To this end the authors considered the optimization problem

$$
\left(\mathrm{P}_{b b, \varepsilon}\right) \min _{g \in L^{2}(\Omega)} J_{b b, \varepsilon}(g)=\frac{1}{2}\left(\int_{0}^{T} \int_{\omega}\left|\varphi_{g}\right| d x d t\right)^{2}+\varepsilon\|g\|_{L^{2}(\Omega)}-\int_{\Omega} y_{d} g d x .
$$


Once again, the problem $\left(\mathrm{P}_{b b, \varepsilon}\right)$ has a unique solution $\bar{g}$, with associated state $\bar{\varphi}$. Taking

$$
\bar{u}(x, t)=\int_{0}^{T} \int_{\omega}|\bar{\varphi}| d x d t \operatorname{sign}(\bar{\varphi}(x, t)), \quad \text { with } \operatorname{sign}(s)=\left\{\begin{array}{cl}
-1 & \text { if } s<0 \\
+1 & \text { if } s>0 \\
{[-1,+1] \quad \text { if } s=0}
\end{array}\right.
$$

the control $\bar{u}$ turns out to be of bang-bang form with a minimum $L^{\infty}$-norm in the class of bounded controls $u$ with associate state satisfying $\left\|y_{u}(T)-y_{d}\right\|_{L^{2}(\Omega)} \leq \varepsilon$.

We refer to [1] for a more detailed presentation of this approach and other related results and, in particular, to the problem of finite-approximate controllability in which, in addition to the approximate controllability property, one also controls exactly the projection of the solution over a finite-dimensional subspace.

The minimization of the $L^{2}$-norm of $\varphi_{g}$ in the problem $\left(\mathrm{P}_{\varepsilon}\right)$ provided the control $\bar{u}$ of minimal $L^{2}$-norm. In problem $\left(\mathrm{P}_{b b, \varepsilon}\right)$, we minimized the $L^{1}$-norm of $\varphi_{g}$, which gave us the control of minimal $L^{\infty}$-norm. Then, it seems natural that if we look for a measure in $Q_{0}$ of minimal norm leading the state to the ball $\bar{B}\left(y_{d}\right)$, then we should study the problem

$$
\left(\mathrm{P}_{\infty, \varepsilon}\right) \quad \min _{g \in L^{2}(\Omega)} J_{\infty, \varepsilon}(g)=\frac{1}{2}\left\|\varphi_{g}\right\|_{C_{0}\left(Q_{0}\right)}^{2}+\varepsilon\|g\|_{L^{2}(\Omega)}-\int_{\Omega} y_{d} g d x .
$$

Let us analyze this control problem. First of all, it is obvious that $J_{\infty, \varepsilon}$ is strictly convex and continuous. Moreover, it is coercive. Indeed, let $\left\{g_{k}\right\}_{k=1}^{\infty} \subset L^{2}(\Omega)$ such that $\left\|g_{k}\right\|_{L^{2}(\Omega)} \rightarrow+\infty$. We will prove that

$$
\lim _{k \rightarrow \infty} \frac{J_{\infty, \varepsilon}\left(g_{k}\right)}{\left\|g_{k}\right\|_{L^{2}(\Omega)}} \geq \varepsilon
$$

To this end, we set $\tilde{g}_{k}=g_{k} /\left\|g_{k}\right\|_{L^{2}(\Omega)}$ and, by taking a subsequence, we can assume that $\tilde{g}_{k} \rightarrow g$ weakly in $L^{2}(\Omega)$. Denote $\varphi_{k}=\varphi_{g_{k}}$ and $\tilde{\varphi}_{k}=\varphi_{\tilde{g}_{k}}$. Then,

$$
\frac{J_{\infty, \varepsilon}\left(g_{k}\right)}{\left\|g_{k}\right\|_{L^{2}(\Omega)}}=\frac{1}{2}\left\|g_{k}\right\|_{L^{2}(\Omega)}\left\|\tilde{\varphi}_{k}\right\|_{C_{0}\left(Q_{0}\right)}^{2}+\varepsilon-\int_{\Omega} y_{d} \tilde{g}_{k} d x
$$

The following two cases may occur:

1.- $\liminf \operatorname{in}_{k \rightarrow \infty}\left\|\tilde{\varphi}_{k}\right\|_{C_{0}\left(Q_{0}\right)}>0$. In this case we obtain immediately that

$$
\frac{J_{\infty, \varepsilon}\left(g_{k}\right)}{\left\|g_{k}\right\|_{L^{2}(\Omega)}} \rightarrow \infty
$$

2.- $\liminf \operatorname{in}_{k \rightarrow \infty}\left\|\tilde{\varphi}_{k}\right\|_{C_{0}\left(Q_{0}\right)}=0$. Now, using the weak* convergence $\tilde{\varphi}_{k} \rightarrow \varphi_{g}$ in $L^{\infty}\left(Q_{0}\right)$ (recall $\left.(7)\right)$, we get by the lower semi-continuity

$$
\left\|\varphi_{g}\right\|_{L^{\infty}\left(Q_{0}\right)} \leq \liminf _{k \rightarrow \infty}\left\|\tilde{\varphi}_{k}\right\|_{L^{\infty}\left(Q_{0}\right)}=0 .
$$


Hence, $\varphi_{g}=0$ in $Q_{0}$. Now, Holmgren Uniqueness Theorem implies that $\varphi_{g} \equiv 0$ in $\Omega \times(0, T)$ and consequently $g=0$. Therefore, $g_{k} \rightarrow 0$ weakly in $L^{2}(\Omega)$ and $\int_{\Omega} y_{d} g_{k} d x \rightarrow 0$ as well. Finally, we have

$$
\liminf _{k \rightarrow \infty} \frac{J_{\infty, \varepsilon}\left(g_{k}\right)}{\left\|g_{k}\right\|_{L^{2}(\Omega)}} \geq \liminf _{k \rightarrow \infty}\left[\varepsilon-\int_{\Omega} y_{d} g_{k} d x\right]=\varepsilon
$$

which concludes the proof.

Let us denote by $\bar{g}_{\infty}$ the solution of $\left(\mathrm{P}_{\infty, \varepsilon}\right)$ and by $\bar{\varphi}_{\infty}$ the associated state. Since we have assumed that $\left\|y_{d}\right\|_{L^{2}(\Omega)}>\varepsilon$, setting $g_{\lambda}=\lambda y_{d}$, it is easy to check that $J_{\infty, \varepsilon}\left(g_{\lambda}\right)<0$ if $\lambda>0$ is small enough. Consequently, we have that $\bar{g}_{\infty} \neq 0$. To write the optimality conditions satisfied by $\bar{g}_{\infty}$, we will use the linear operator $A^{*} \in \mathcal{L}\left(L^{2}(\Omega), C_{0}\left(Q_{0}\right)\right)$ introduced in $\S 3$. Then, $J_{\infty, \varepsilon}$ can be expressed in the form

$$
J_{\infty, \varepsilon}(g)=\frac{1}{2}\left\|A^{*} g\right\|_{C_{0}\left(Q_{0}\right)}^{2}+\varepsilon\|g\|_{L^{2}(\Omega)}-\int_{\Omega} y_{d} g d x .
$$

Then, it holds $0 \in \partial J_{\infty, \varepsilon}\left(\bar{g}_{\infty}\right)$, which implies the existence of an element $\bar{u}_{\infty} \in$ $\partial\|\cdot\|_{C_{0}\left(Q_{0}\right)}\left(\bar{\varphi}_{\infty}\right)$, where $\bar{\varphi}_{\infty}=\varphi_{\bar{g}_{\infty}}$, such that

$$
0=\left\|\bar{\varphi}_{\infty}\right\|_{C_{0}\left(Q_{0}\right)} A \bar{u}_{\infty}+\frac{\varepsilon}{\left\|\bar{g}_{\infty}\right\|_{L^{2}(\Omega)}} \bar{g}_{\infty}-y_{d} .
$$

If $\bar{y}_{\infty}$ denotes the solution of (1) corresponding to $\bar{u}_{\infty}$, then $A \bar{u}_{\infty}=\bar{y}_{\infty}(T)$; see $\S 3$. Then the above equality can be rewritten

$$
\left\|\bar{\varphi}_{\infty}\right\|_{C_{0}\left(Q_{0}\right)} \bar{y}_{\infty}(T)-y_{d}=-\frac{\varepsilon}{\left\|\bar{g}_{\infty}\right\|_{L^{2}(\Omega)}} \bar{g}_{\infty}
$$

On the other hand, $\bar{u}_{\infty} \in \partial\|\cdot\|_{C_{0}\left(Q_{0}\right)}\left(\bar{\varphi}_{\infty}\right)$ implies by definition

$$
\int_{Q_{0}}\left(z-\bar{\varphi}_{\infty}\right) d \bar{u}_{\infty}+\left\|\bar{\varphi}_{\infty}\right\|_{C_{0}\left(Q_{0}\right)} \leq\|z\|_{C_{0}\left(Q_{0}\right)} \forall z \in C_{0}\left(Q_{0}\right) .
$$

These inequalities are equivalent to

$$
\left\|\bar{\varphi}_{\infty}\right\|_{C_{0}\left(Q_{0}\right)}=\int_{Q_{0}} \bar{\varphi}_{\infty} d \bar{u}_{\infty} \quad \text { and } \quad\left\|\bar{u}_{\infty}\right\|_{M\left(Q_{0}\right)}=1
$$

Finally, if we set

$$
(\bar{g}, \bar{\varphi})=-\frac{1}{\left\|\bar{\varphi}_{\infty}\right\|_{C_{0}\left(Q_{0}\right)}}\left(\bar{g}_{\infty}, \bar{\varphi}_{\infty}\right) \quad \text { and } \quad(\bar{u}, \bar{y})=\left\|\bar{\varphi}_{\infty}\right\|_{C_{0}\left(Q_{0}\right)}\left(\bar{u}_{\infty}, \bar{y}_{\infty}\right),
$$

then we get from (16) and (17) that $(\bar{g}, \bar{\varphi}, \bar{u}, \bar{y})$ satisfies (9)-(12) and consequently $\bar{u}$ is the solution of $(\mathrm{P})$.

We have proved that the solution $\bar{\varphi}_{\infty}$ of $\left(\mathrm{P}_{\infty, \varepsilon}\right)$ also gives the solution of $(\mathrm{P})$ by the formula $\bar{u} \in\left\|\bar{\varphi}_{\infty}\right\|_{C_{0}\left(Q_{0}\right)} \partial\|\cdot\|_{C_{0}\left(Q_{0}\right)}\left(\bar{\varphi}_{\infty}\right)$. Analogously, the solution $\bar{\varphi}$ 
of problem $\left(\mathrm{P}^{*}\right)$, studied in $\S 3$, provided the solution of $(\mathrm{P})$ by the formula $\bar{u} \in$ $-\partial\|\cdot\|_{C_{0}\left(Q_{0}\right)}(\bar{\varphi})$. If we compare the functionals $J_{\infty, \varepsilon}$ and $\mathcal{G}$, whose minimization lead to $\bar{\varphi}_{\infty}$ and $\bar{\varphi}$, respectively, we remark two differences. One is the different sign in the integral $\int_{\Omega} y_{d} g d x$, which justifies the different signs in the previous formulas for $\bar{u}$. The second and most important difference comes from the terms involving $\varphi_{g}$ : $(1 / 2)\left\|\varphi_{g}\right\|_{C_{0}\left(Q_{0}\right)}^{2}$ and $I_{B}\left(\varphi_{g}\right)$. It is interesting to observe that the function $I_{B}\left(\varphi_{g}\right)$ coincides with $J^{*}\left(A^{*} g\right)$, as proved in $\S 3$, and $J_{\infty, \varepsilon}\left(\varphi_{g}\right)$ coincides with $F^{*}\left(A^{*} g\right)$, where $F: M\left(Q_{0}\right) \longrightarrow \mathbb{R}$ is defined by $F(u)=(1 / 2)\|u\|_{M\left(Q_{0}\right)}^{2}$. Therefore, the problem $\left(\mathrm{P}_{\infty, \varepsilon}\right)$ is, except for the sign before the integral involving $y_{d}$, the dual of the problem

$$
(\tilde{\mathrm{P}}) \quad\left\{\begin{array}{l}
\min F(u)=\frac{1}{2}\|u\|_{M\left(Q_{0}\right)}^{2}, \\
\left(u, y_{u}(T)\right) \in M\left(Q_{0}\right) \times \bar{B}_{\varepsilon}\left(y_{d}\right) .
\end{array}\right.
$$

It is obvious that $(\mathrm{P})$ and $(\tilde{\mathrm{P}})$ are essentially the same. Consequently, the dual problems $\left(\mathrm{P}^{*}\right)$ and $\left(\mathrm{P}_{\infty, \varepsilon}\right)$ are very close. Indeed, the problem $\left(\mathrm{P}^{*}\right)$ can be transformed into $\left(\mathrm{P}_{\infty, \epsilon}\right)$ by a simple scaling. This is not surprising. Indeed, the solution $\bar{g}_{\infty}$ of $\left(\mathrm{P}_{\infty, \varepsilon}\right)$ is the solution of

$$
\left\{\begin{array}{l}
\min \varepsilon\|g\|_{L^{2}(\Omega)}-\int_{\Omega} y_{d} g d x, \\
g \in L^{2}(\Omega) \text { and }\left\|\varphi_{g}\right\|_{C_{0}\left(Q_{0}\right)} \leq\left\|\bar{\varphi}_{\infty}\right\|_{C_{0}\left(Q_{0}\right)} .
\end{array}\right.
$$

It is enough to divide $g$ by $-\left\|\bar{\varphi}_{\infty}\right\|_{C_{0}\left(Q_{0}\right)}$ to obtain the problem $\left(\mathrm{P}^{*}\right)$, and then $\bar{g}=-g_{\infty} /\left\|\bar{\varphi}_{\infty}\right\|_{C_{0}\left(Q_{0}\right)}$.

\section{The elliptic case}

In this section, we consider the following elliptic version of the control problem $(\mathrm{P})$ :

$$
\text { (E) }\left\{\begin{array}{l}
\min J(u)=\|u\|_{M(\Omega)}, \\
\left(u, y_{u}\right) \in M(\Omega) \times \bar{B}_{\varepsilon}\left(y_{d}\right),
\end{array}\right.
$$

where $M(\Omega)=C_{0}(\Omega)^{*}$ is the space of real and regular Borel measures in $\Omega$, $B_{\varepsilon}\left(y_{d}\right)$ is the ball of radious $\varepsilon$ in $L^{2}(\Omega)$ with center on $y_{d}$, and $y_{u}$ is the solution of

$$
\left\{\begin{array}{rll}
-\Delta y & =u & \text { in } \Omega, \\
y & =0 & \text { on } \Gamma .
\end{array}\right.
$$

The results we shall present are also valid for more general second order elliptic operator with Lipschitz continuous coefficients $a_{i j} \in C(\bar{\Omega})$. Indeed, by using the coefficient freezing method, the elliptic operator is locally reduced to the Laplace operator, which allows us to get the regularity results we need and the Lipschitz regularity of the coefficients ensures the key unique continuation property.

For technical reasons we limit our discussion to the cases $1 \leq n \leq 3$. Indeed, observe that given a measure $u \in M(\Omega)$, there exists a unique solution $y$ of (18) 
belonging to $W_{0}^{1, p}(\Omega)$ for every $1 \leq p<\frac{n}{n-1}$. Moreover, the following inequality holds

$$
\|y\|_{W_{0}^{1, p}(\Omega)} \leq C_{p}\|u\|_{M(\Omega)}
$$

see, for instance, [17] and [18]. Since $W_{0}^{1, p}(\Omega) \subset L^{2}(\Omega)$ for very $\frac{2 n}{n+2} \leq p<\frac{n}{n-1}$, the problem $(\mathrm{E})$ is well defined in $M(\Omega)$. In view of this, in dimensions $n \geq 4$ similar problems can be considered but by taking $B_{\varepsilon}\left(y_{d}\right)$ to be the ball in a suitable $L^{p}(\Omega)$ space, with $p$ depending on the dimension $n$.

As for the problem $(\mathrm{P})$, hereafter we assume that $\left\|y_{d}\right\|_{L^{2}(\Omega)}>\varepsilon$, otherwise the solution of the problem $(\mathrm{E})$ is $\bar{u}=0$. Concerning the solvability of $(\mathrm{E})$, we have the following result.

Theorem 5. The control problem $(\mathrm{P})$ has a unique solution $\bar{u}$. Moreover, the associated state $\bar{y}$ satisfies $\left\|\bar{y}-y_{d}\right\|_{L^{2}(\Omega)}=\varepsilon$.

Proof. The existence of $u$ such that the state $y$ belongs to $\bar{B}_{\varepsilon}\left(y_{d}\right)$ is obvious by a density argument and due to the fact that $-\Delta$ is an isomorphism from $H^{2}(\Omega) \cap H_{0}^{1}(\Omega)$ into $L^{2}(\Omega)$. Then, the existence of a solution can be easily proved by taking a minimizing sequence and using the continuity of $u \in M(\Omega) \rightarrow y_{u} \in$ $L^{2}(\Omega)$; see (19). Finally, the proof of the uniqueness of solution follows the steps of the proof of the uniqueness of $\bar{y}_{T}$ in Theorem 2. To this end we use the injectivity of the control-to-state mapping.

The analogue of Theorem 3 also holds for problem (E) as follows.

Theorem 6. Let $\bar{u} \in M(\Omega)$ such that $\bar{y} \in \bar{B}_{\varepsilon}\left(y_{d}\right)$, where $\bar{y}$ is the state associated to $\bar{u}$. Then, $\bar{u}$ is the solution of problem (E) if and only if there exist two unique elements $\bar{g} \in L^{2}(\Omega)$ and $\bar{\varphi} \in W_{0}^{1, s}(\Omega)$, for $n<s \leq n+\delta_{\Omega}$, for some $\delta_{\Omega}>0$, such that

$$
\begin{aligned}
& \int_{\Omega} \bar{g}(x)(y(x)-\bar{y}(x)) d x \leq 0 \quad \forall y \in \bar{B}_{\varepsilon}\left(y_{d}\right), \\
& \left\{\begin{array}{rll}
-\Delta \bar{\varphi} & =\bar{g} \text { in } \Omega, \\
\bar{\varphi} & =0 \text { on } \Gamma,
\end{array}\right. \\
& \|\bar{u}\|_{M(\Omega)}=-\int_{\Omega} \bar{\varphi} d \bar{u}, \\
& \|\bar{\varphi}\|_{C_{0}(\Omega)}=1 \text {. }
\end{aligned}
$$

Furthermore, there exists a real number $\bar{\lambda}>0$ such that $\bar{g}=\bar{\lambda}\left(\bar{y}-y_{d}\right)$.

Proof. The proof follows the same steps as in Theorem 3. Let us mention the differences. First, we observe that the regularity $\bar{\varphi}$ follows from [18]. It is proved in [18] that $\delta_{\Omega}>2$ for $n=2$ and $\delta_{\Omega}>0$ if $n=3$. For $n=3$ we also assume that $\delta_{\Omega} \leq 3$ due to the necessary embedding $L^{2}(\Omega) \subset W^{-1, s}(\Omega)$. In the case of a $C^{1}$ boundary $\Gamma, \varphi \in W_{0}^{1, s}(\Omega)$ for all $2<s<\infty$ if $n=2$ and for $s=6$ if $n=3$. In any case, since $s>n, \bar{\varphi} \in W_{0}^{1, s}(\Omega) \subset C_{0}(\Omega)$. Now, we consider the linear mapping $A \in \mathcal{L}\left(M(\Omega), L^{2}(\Omega)\right)$, defined by $A u=y_{u}$ solution of (18). The 
continuity of $A$ follows from (19). Then, the problem (E) can be reformulated as the minimization of the functional $\mathcal{J}: M(\Omega) \longrightarrow \mathbb{R}$ defined by

$$
\mathcal{J}(u)=J(u)+I_{\bar{B}_{\varepsilon}\left(y_{d}\right)}(A u) .
$$

Finally, we have to prove the identity

$$
\left\langle A^{*} \bar{g}, u\right\rangle=\int_{\Omega_{0}} \bar{\varphi} d u \quad \forall u \in M(\Omega) .
$$

Since $y_{u} \in W_{0}^{1, p}(\Omega)$ for every $p<\frac{n}{n-1}$, we can take $p$ close enough to $\frac{n}{n-1}$ such that $n<s=p^{\prime} \leq n+\delta_{\Omega}$. Therefore, we can multiply the equation (18) by $\bar{\varphi}$ and make an integration by parts to get with (21)

$$
\int_{\Omega} \bar{\varphi} d u=\int_{\Omega} \nabla y_{u} \nabla \bar{\varphi} d x=\int_{\Omega} \bar{g} y_{u} d x=\int_{\Omega} \bar{g} A u d x=\left\langle A^{*} \bar{g}, u\right\rangle,
$$

which proves (24).

Corollary 3. Let $\bar{u}=\bar{u}^{+}-\bar{u}^{-}$be the Jordan decomposition of the measure $\bar{u}$, then the following inclusions hold

$$
\left\{\begin{array}{l}
\operatorname{supp}\left(\bar{u}^{+}\right) \subset\{x \in \Omega: \bar{\varphi}(x)=-1\}, \\
\operatorname{supp}\left(\bar{u}^{-}\right) \subset\{x \in \Omega: \bar{\varphi}(x)=+1\} .
\end{array}\right.
$$

This corollary is deduced from (22) and (23) as in the proof of Corollary 1. Concerning the support of the measure $\bar{u}$, we can say that it is reduced to a finite set of points in many situations, while, in others, for instance, the set where $\bar{\varphi}(x)=+1$ (respectively, -1 ) may be a curve or a hypersurface of dimension $n-1$.

Problem (E) is very close to the one studied by Clason and Kunisch [7]

$$
\left(\mathrm{P}_{\alpha}\right)\left\{\begin{array}{l}
\min J_{\alpha}(u)=\frac{1}{2}\left\|y_{u}-y_{d}\right\|_{L^{2}(\Omega)}^{2}+\alpha\|u\|_{M(\Omega)}, \\
u \in M(\Omega),
\end{array}\right.
$$

where $\alpha>0$. Indeed, let us assume that $u_{\alpha}$ is the solution of $\left(\mathrm{P}_{\alpha}\right)$ with $y_{\alpha}$ and $\varphi_{\alpha}$ as associated state and adjoint state, respectively. Let us denote $\varepsilon_{\alpha}=\left\|y_{\alpha}-y_{d}\right\|_{L^{2}(\Omega)}^{2}$, then it is immediate that $u_{\alpha}$ is the solution of (E) for $\varepsilon=\varepsilon_{\alpha}$. This follows from the fact that the state $\bar{y}$ associated to the solution $\bar{u}$ of $(\mathrm{E})$ satisfies $\left\|\bar{y}-y_{d}\right\|_{L^{2}(\Omega)}=\varepsilon$. Moreover, as proved in [7], $\left(u_{\alpha}, y_{\alpha}, \varphi_{\alpha}\right)$ satisfies

$$
\begin{aligned}
\left\{\begin{array}{rlr}
-\Delta \varphi_{\alpha} & =y_{\alpha}-y_{d} & \text { in } \Omega, \\
\varphi_{\alpha} & =0 & \text { on } \Gamma,
\end{array}\right. \\
\left\|u_{\alpha}\right\|_{M(\Omega)}=-\frac{1}{\alpha} \int_{\Omega} \varphi_{\alpha} d \bar{u}, \\
\left\|\varphi_{\alpha}\right\|_{C_{0}(\Omega)}=\alpha .
\end{aligned}
$$


Comparing this system with (21)-(23), we deduce that $\bar{\varphi}=\frac{1}{\alpha} \varphi_{\alpha}$ and $\bar{\lambda}=\frac{1}{\alpha}$. Conversely, if $\bar{u}$ is the solution of (E) and we take $\bar{\lambda}$ as in Theorem 6 , we deduce that $\bar{u}$ is the solution of problem $\left(\mathrm{P}_{\alpha}\right)$ for $\alpha=1 / \bar{\lambda}$. Indeed, it is enough to observe that $(\bar{u}, \bar{y}, \bar{\varphi} / \bar{\lambda})$ satisfies the optimality system $(26)-(28)$.

Although the solution $\bar{u}$ of $(\mathrm{E})$ is a measure with possibly a support not reduced to a finite number of points, its numerical approximation can be carried out in a efficient way by a linear combination of Dirac measures. The reader is referred to [8] for this issue.

Concerning the dual problem of $(\mathrm{E})$, we can proceed as in $\S 3$ to deduce that it is formulated as follows

$$
\left(\mathrm{E}^{*}\right)\left\{\begin{array}{l}
\sup \left\{-\varepsilon\|g\|_{L^{2}(\Omega)}-\left(y_{d}, g\right)_{L^{2}(\Omega)}\right\} \\
\left(g, \varphi_{g}\right) \in L^{2}(\Omega) \times B
\end{array}\right.
$$

where $B$ is the unit closed ball of $C_{0}(\Omega)$ centered at 0 and $\varphi_{g}$ is the solution of the Dirichlet problem

$$
\left\{\begin{array}{rll}
-\Delta \varphi & =g & \text { in } \Omega \\
\varphi & =0 & \text { on } \Gamma .
\end{array}\right.
$$

Analogous comments to those of $\S 4$ can be translated to the control problem (E) and its dual $\left(\mathrm{E}^{*}\right)$. In particular, the adjoint methodology for the approximate controllability of the heat equation, based on the minimization of the functional $J_{\infty, \varepsilon}$, can be adapted to the present context. Indeed, it suffices to minimize the functional

$$
J_{\infty, \varepsilon}(g)=\frac{1}{2}\left\|\varphi_{g}\right\|_{C_{0}(\Omega)}^{2}+\varepsilon\|g\|_{L^{2}(\Omega)}-\int_{\Omega} y_{d} g d x,
$$

for $g \in L^{2}(\Omega)$ where $\varphi_{g}$ is the solution of the adjoint equation (5). The minimizer $\tilde{g} \in L^{2}(\Omega)$ exists and is unique and it leads to an approximate control, localized on the set where the corresponding adjoint state $\tilde{\varphi}$ achieves extremal values $\pm\left\|\tilde{\varphi}_{g}\right\|_{C_{0}(\Omega)}$.

Similar issues can be treated in the case where the control is localized in a subset on $\Omega$. We refer to [19] for the analysis of these problems in the $L^{2}$ setting. The adaptation of the techniques of the present paper to that setting is an interesting open problem.

\section{Further comments and open problems}

The methods and results of the present paper can be extended to various other problems. Let us mention some of them:

- Boundary control for the heat equation: Similar results hold in the context of the boundary control of the heat equation for various boundary conditions, in particular of Dirichlet and Neumann type. 
- Null control of the heat equation: The same techniques can be applied in the context of the null control of the heat equation in which the goal is to drive the solution to the null equilibrium, both in the context of internal or boundary control. We refer to [1] for a recent survey on the state of the art in what concerns the problem of null control of the heat equation in the classical context of smooth and bang-bang controls.

A number of interesting issues are still widely open and need to be investigated.

- Wave equations: The same problems make sense for the wave equation. But this time, optimal measures can not be guaranteed to be localized on narrow sets. Indeed, the sets where the solutions of the adjoint wave equation achieve extremal values ( \pm its $L^{\infty}$-norm) can be arbitrary. Similar phenomena occur in what concerns the bang-bang property that fails to hold for the wave equation (see [1] and [20]).

- Semilinear heat equations: There is an extensive literature on the existence of controls of minimal $L^{2}$-norm and of bang-bang form for the null and approximate control of the semilinear heat equation (see [1]). Most often controls are built by fixed point arguments, by means of a careful analysis of the dependence of controls of the heat equation with a potential. The problem of whether optimal control measures are supported on a finite number of points is open in that case due, mainly, to the fact that such a property, at least along the proof developed in the present article needs the potential to be analytic.

\section{References}

[1] E. Zuazua, Controllability and observability of partial differential equations: some results and open problems, in: C. M. Dafermos, E. Feireisl (Eds.), Handbook of differential equations: evolutionary equations. Vol. III, Handb. Differ. Equ., Elsevier/NorthHolland, Amsterdam, 527-621, doi:10.1016/S1874-5717(07)80010-7, URL http://dx.doi.org/10.1016/S1874-5717(07)80010-7, 2006.

[2] J.-L. Lions, Remarks on approximate controllability, J. Analyse Math. 59 (1992) 103-116.

[3] E. Casas, R. Herzog, G. Wachsmuth, Optimality conditions and error analysis of semilinear elliptic control problems with $L^{1}$ cost functional, SIAM J. Optim. (to appear).

[4] R. Herzog, G. Stadler, G. Wachsmuth, Directional Sparsity in Optimal Control of Partial Differential Equations, SIAM J. Control Optim. 50 (2) (2012) 943-963.

[5] G. Stadler, Elliptic Optimal Control Problems with $L^{1}$-Control Cost and Applications for the Placement of Control Devices, Comp. Optim. Appls. 44 (2) (2009) 159-181. 
[6] G. Wachsmuth, D. Wachsmuth, Convergence and Regularisation Results for Optimal Control Problems with Sparsity Functional, ESAIM Control Optim. Calc. Var. 17 (3) (2011) 858-886.

[7] C. Clason, K. Kunisch, A Duality-Based Approach to Elliptic Control Problems in Non-Reflexive Banach Spaces, ESAIM Control Optim. Calc. Var. 17 (1) (2011) 243-266.

[8] E. Casas, C. Clason, K. Kunisch, Approximation of elliptic control problems in measure spaces with sparse solutions, SIAM J. Control Optim. (to appear).

[9] W. Rudin, Real and Complex Analysis, McGraw-Hill, London, 1970.

[10] E. Casas, Pontryagin's principle for state-constrained boundary control problems of semilinear parabolic equations, SIAM J. Control Optim. 35 (4) (1997) 1297-1327.

[11] J. Raymond, H. Zidani, Pontryagin's principle for state-constrained control problems governed by parabolic equations with unbounded controls, SIAM J. Control Optim. 36 (6) (1998) 1853-1879.

[12] C. Fabre, J.-P. Puel, E. Zuazua, in: Control and optimal design of distributed parameter systems (Minneapolis, MN, 1992), volume 70 of IMA Vol. Math. Appl., Springer, New York, 1995, pp. 73-91.

[13] O. Ladyzhenskaya, V. Solonnikov, N. Ural'tseva, Linear and Quasilinear Equations of Parabolic Type, American Mathematical Society, 1968.

[14] I. Ekeland, R. Temam, Convex Analysis and Variational Problems, NorthHolland-Elsevier, New York, 1976.

[15] L.C. Evans, Partial differential equations, Graduate Studies in Mathematics, 19, American Mathematical Society, Providence, RI, 1998.

[16] C. Fabre, J. P. Puel, E. Zuazua, Approximate controllability for the semilinear heat equation, Proc. Roy. Soc. Edinburgh 125A (1995) 31-61.

[17] E. Casas, Control of an elliptic problem with pointwise state constraints, SIAM J. Control Optim. 24 (6) (1986) 1309-1318.

[18] D. Jerison, C. Kenig, The inhomogeneous Dirichlet problem in Lipschitz domains, J. Funct. Anal. 130 (1995) 161-219.

[19] A. Osses, J.-P. Puel, On the controllability of the Laplace equation observed on an interior curve, Revista Matemática Complutense 1 (2) (1998) 403441 .

[20] M. Gugat, G. Leugering, $L^{\infty}$-norm minimal control for the wave equation: on the weakness of the bang-bang principle, ESAIM: COCV 14 (2008) 254283 . 